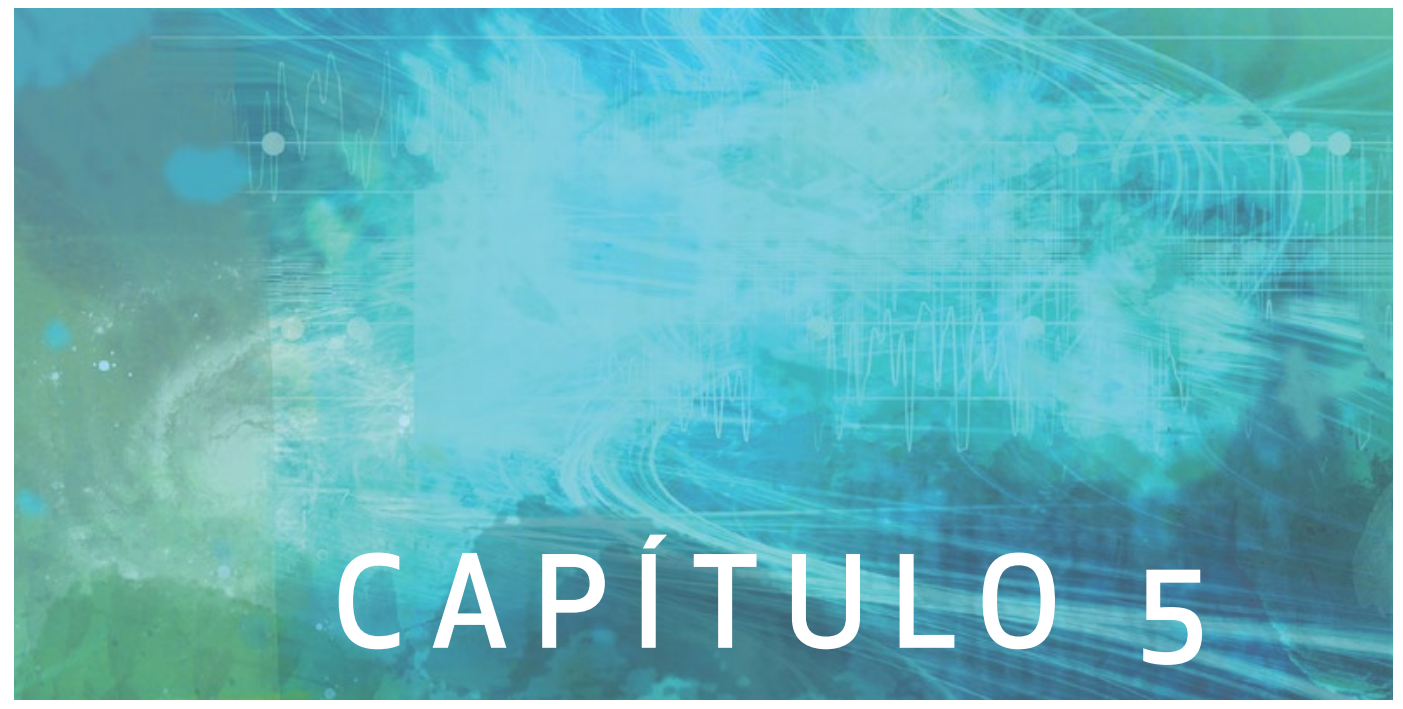





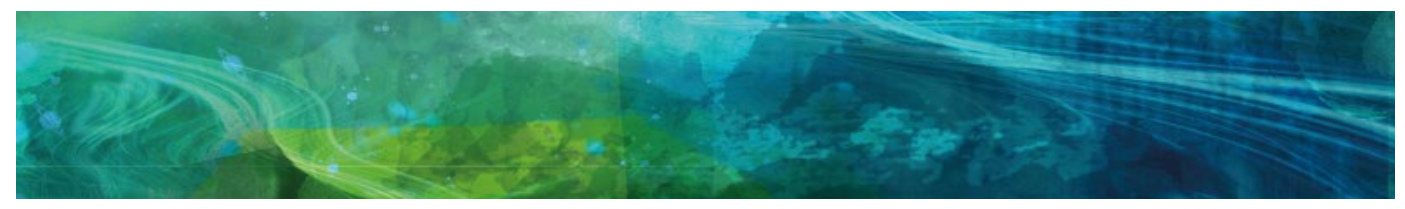

\section{Propuesta de un modelo de competitividad para el emprendimiento social}

Juan Camilo Vega Guerrero ${ }^{8}$

8 Estudiante de doctorado, Universidad de Celaya, México. Magister en Administración y Especialista en Estadística, Universidad Nacional de Colombia. Administrador de Empresas. Docente de la UNAD. Contacto: juan.vega@unad.edu.co 


\section{Resumen}

En el presente capítulo se identifican elementos de competitividad para emprendimientos sociales que difieren con los componentes que integran los modelos de competitividad para empresa tradicionales. Se resaltan, ente otros: el valor social, el capital social, y la innovación como estrategia para la sostenibilidad de la organización.

Palabras clave: emprendimiento social, empresa social, competitividad organizacional, emprendedor social

\section{Introducción}

El emprendimiento social es un factor relevante para el desarrollo económico y la generación efectiva de puestos de trabajo en países subdesarrollados` (Guzmán y Trujillo, 2008; Harding, 2004). En este sentido, el objetivo de este capítulo es caracterizar una propuesta de modelo de competitividad para emprendimientos sociales con el fin de establecer los compontes que deben ser sujeto de medición para mejorar la competitividad de este tipo de emprendimiento. En la medida que difiere con el emprendimiento tradicional cuyo propósito es el de aprovechar oportunidades de mercado y generar beneficios particulares o para sus propietarios.

El propósito del capítulo está enmarcado dentro del proyecto de investigación: modelo de formación en emprendimiento social para programas académicos de administración de empresas y negocios, con modalidad de educación a distancia en Colombia, de la Escuela de Ciencias Administrativas, Contables, Económicas y de Negocios (ECACEN).

9 Colombia es un país considerado como un país en desarrollo, de ingreso medio alto y su renta per cápita está en un 70 \% por debajo del promedio de la OCDE y por debajo de muchos otros mercados emergentes (OCDE, 2013). 
Para el diseño de la propuesta del modelo, el capítulo se desarrolla en los siguientes apartes: concepto y tipos empresas sociales, conceptos y modelos de competitividad, emprendimiento social y perfil de emprendedor social

Al finalizar el capítulo se caracterizan los compontes del modelo de competitividad para emprendimiento social, definidos como capital social y administración, gestión de recursos, talento humano, valor social y sostenibilidad e innovación. Finalmente se concluye que los aspectos que evidencian competitividad de los emprendimientos sociales son: la generación de capital y el valor social.

\section{Empresas sociales}

El concepto de empresa social nace en Italia (1990), en la revista Impresa Sociale, y ha venido transformándose. Uno de los primeros conceptos es el de l'émergence des Enterprises Sociales (EMES), concepto de origen europeo, y se direcciona sobre dos dimensiones, una de orden económico y la otra de tipo social. La dimensión económica se enfoca sobre:

- La producción de bienes y servicios para su comercialización o venta.

- Nivel significativo de autonomía.

- Alto riesgo económico.

- Mínima mano de obra remunerada.

De otro lado, la dimensión social se enfoca en:

- Objetivo explícito de beneficiar a la comunidad.

- Iniciativas gestionadas por un grupo de ciudadanos.

- La propiedad del capital no es el factor relevante para la toma de decisiones.

- Intervienen diferentes grupos de interés.

- Distribución limitada de los beneficios. 


\section{Adicionalmente a los aspectos mencionados, el Comité Económico y Social Europeo incluyen como aspectos que caracterizan a este tipo de organiza- ciones la inclusión social y el emprendimiento social (Villajos, et al., 2012). A continuación, se presentan definiciones de interés sobre la empresa social.}

Tabla 1. Definiciones sobre la empresa social.

\section{Definición Autor}

Organizaciones privadas cuyo propósito es resolver problemas sociales o servir a personas en condición de vulnerabilidad o marginadas. También buscan abastecer bienes socialmente importantes que no son suministrados desde las entidades gubernamentales o el sector privado.

Dees (1994); citado en Mair y

Martí, (2006).

Las organizaciones buscan propósitos que no solamente se miden por la generación de utilidad o la participación en el sector económico.

Las empresas sociales son gestadas por ciudadanos que pretenden proveer una diversidad de servicios sin restricciones para la comunidad.

Organizaciones particulares, diferentes a la empresa Laville y Nyssens, (2001). privada y la pública, donde el poder no se soporta en la propiedad de los recursos o bienes de capital.

Propuestas voluntarias originadas desde grupos de ciudadanos debido a una demanda insatisfecha en la comunidad generada por externalidades negativas debido a una crisis económica o de dificultades del Estado. Para lo cual, crean una estructura organizacional Vidal y Claver, (2003). no lucrativa para desarrollar actividades que permitan satisfacer esta demanda insatisfecha.

Las empresas sociales son una nueva forma de empresa, con propósitos sociales y que compiten en el mercado.

Spear y Bidet, (2005).

Son organizaciones que se ubican en el sector no lucrativo, en donde la misión social es esencial, adoptando modelos de negocio innovadores para resolver un problema en la Galera y Borzaga, (2009). comunidad y crear valor social.

Organizaciones con una misión social y orientada a la generación de recurso para su sostenibilidad.

Palacios, (2010).

Fuente: Elaboración propia a partir de los autores citados 
En este contexto se identifican dos corrientes de pensamiento. La anglosajona, donde una empresa privada - fines lucrativos-, si presta servicios a comunidades vulnerables, se podría reconocer como empresa social. En el otro enfoque, el europeo, el caso de la empresa mencionada no podría ser catalogada como empresa social (Villajos y Sanchis, 2013). Por lo tanto, y en consecuencia con la variedad de definiciones, es útil identificar los factores que convergen en las empresas sociales.

1). Capital social: capacidad de fortalecer las relaciones dentro y alrededor de la organización con el propósito de movilizar recursos individuales, y la movilización de la comunidad local o intervenida (Bacchiega y Borzaga, 2001). Este aspecto permite disminuir los costos de intermediación-transacción y de producción en la medida que la empresa logra integrar los grupos de interés para resolver problemas del contexto que las instituciones gubernamentales no logran resolver (Laville y Nyssens, 2001).

2). Diversidad de recursos: la fuente de financiación proviene de diferentes entidades, no solo públicas. Es decir que la organización debe diversificar en la búsqueda de fuentes para su sostenibilidad. En este punto también son fuente de recursos, entre otros, la participación de talento humano con mecanismo, el voluntariado y otros (Vidal, 2001; Defourny, 2001; Salinas y Rubio, 2001; Defourny, 2004; Spear y Bidet, 2005; Nyssens, 2006).

3). Diferentes formas jurídicas de conformación: existen diferentes formas de conformar empresas sociales según el país de origen. Predomina la conformación de tipo asociativo (Defourny, 2001; Vidal y Claver, 2003; Spear y Bidet, 2005; Spear, Cornforth y Aken, 2009).

4). Diversos tipos de beneficiarios: en las empresas sociales existen diferentes grupos de interés a los cuales la empresa social debe dar respuesta: trabajadores, clientes, propietarios, comunidad 
entre otros. Así mismo prima el beneficio del colectivo sobre el beneficio individual (Lavilley Nyssens, 2001).

5). Diferentes métodos de organización: la manera de estructurar la organización depende en gran medida de la forma como se permite la participación de la comunidad (Galera y Borzaga, 2009, p. 217) y su interacción con los diferentes grupos de interés para promover el desarrollo económico y social de la comunidad intervenida.

6). Variedad de objetivos: los objetivos de estas empresas se deben conjugar en dos direcciones, a saber, los propósitos económicos y sociales (Nyssens, 2006; Galera y Borzaga, 2009). Los primeros permiten la viabilidad y sostenibilidad de la organización y los segundos se enfocan en dar repuestas a las problemáticas o necesidades de la comunidad intervenida.

7). Valor social sostenible: Ios beneficios obtenidos no son distribuidos entre los accionistas o propietarios de la empresa sino reinvertidos en la empresa para fomentar el desarrollo económico y social en la comunidad o contexto intervenido (Villajos y Sanchis, 2013).

8). Especialización: los procesos de gestión en la comunidad obligan a las empresas sociales a ser eficientes para ser viables, al igual que las empresas de capitales (Salinas y Rubio, 2001, p. 88).

\section{Tipos de empresas sociales}

Según los propósitos y origen de los recursos se pueden establecer los siguientes tipos de empresas sociales. 
Tabla 2. Tipos de empresas sociales.

\begin{tabular}{|c|c|c|c|}
\hline Categoría & $\begin{array}{l}\text { Origen de los } \\
\text { recursos }\end{array}$ & Propósito social & Autores \\
\hline Mutuales & $\begin{array}{l}\text { Los recursos provienen } \\
\text { de los miembros } \\
\text { que conforman la } \\
\text { organización. }\end{array}$ & $\begin{array}{l}\text { Satisfacer las necesidades } \\
\text { de los miembros que } \\
\text { conforman la empresa } \\
\text { (salud, alimentación, } \\
\text { crédito) }\end{array}$ & $\begin{array}{l}\text { Neck, Brush y Allen, (2009); } \\
\text { Spear, Cornforth y Aiken, } \\
\text { (2009); Díaz-Foncea y } \\
\text { Marcuello, (2012) }\end{array}$ \\
\hline Externas & $\begin{array}{l}\text { Los recursos provienen } \\
\text { de un entidad externa } \\
\text { que apadrina o } \\
\text { auspicia a la entidad }\end{array}$ & $\begin{array}{l}\text { Solucionar, paliar } \\
\text { o disminuir una } \\
\text { problemática social. }\end{array}$ & $\begin{array}{l}\text { Neck, Brush y Allen, (2009); } \\
\text { Terjesen et Al, (2011); } \\
\text { Spear, Cornforth y Aiken, } \\
\text { (2009); Díaz-Foncea y } \\
\text { Marcuello, (2012) }\end{array}$ \\
\hline Híbridas & $\begin{array}{l}\text { El trabajo en red entre } \\
\text { una entidad privada } \\
\text { y distintos grupos de } \\
\text { interés. }\end{array}$ & $\begin{array}{l}\text { Satisfacer las necesidades } \\
\text { de los miembros de una } \\
\text { comunidad que no son } \\
\text { cubiertas por el estado o } \\
\text { las empresas de capital. }\end{array}$ & $\begin{array}{l}\text { Neck, Brush y Allen, (2009). } \\
\text { Terjesen et Al, (2011); } \\
\text { Spear, Cornforth y Aiken, } \\
\text { (2009); Díaz-Foncea y } \\
\text { Marcuello, (2012) }\end{array}$ \\
\hline
\end{tabular}

Fuente: Elaboración propia a partir de los autores citados

\section{Competitividad}

El concepto de competitividad se ha desarrollado desde siglos pasados, con autores como David Hume, Adam Smith, David Ricardo y Gottfried Haberler. Ellos se focalizan en aspectos relacionados con el intercambio a nivel internacional para mejorar la riqueza y competitividad de las naciones (Pat, et al., 2009).

En el presente, el desarrollo del término se enfoca en tres niveles: a nivel de país, de un sector económico, de una rama de la industria y de una empresa. Resaltando que, para un variado grupo de investigadores, son las empresas las que compiten y no las naciones, por ello en el escenario de competitividad intervienen, aparte de las empresas, varios actores como el sector productivo, el gobierno, y los usuarios o clientes (Montoya, et al., 2012).

Para el contexto de discusión nos centramos en la dimensión de competitividad empresarial. Bajo este parámetro se presentan a continuación definiciones al respecto para identificar elementos que caracterizan la competitividad empresarial. 
Tabla 3. Definiciones de competitividad empresarial.

\begin{tabular}{ll}
\multicolumn{1}{c}{ Definición } & \multicolumn{1}{c}{ Autor } \\
\hline $\begin{array}{l}\text { Es la capacidad de una empresa para alcanzar una posición } \\
\text { competitiva favorable que permita la obtención de un } \\
\text { desempeño superior a las empresas de la competencia. }\end{array}$ & Rubio y Aragón (2006) \\
\hline $\begin{array}{l}\text { La competitividad empresarial es consecuencia de la ventaja } \\
\text { competitiva que tiene una organización soportada en sus }\end{array}$ & Abdel y Romo (2004) \\
métodos de producción y de organización con respecto a & \\
competidores del sector económico. & \\
\hline $\begin{array}{l}\text { La competitividad de la empresa esta correlacionada con: } \\
\text { la competitividad del país, la apertura y acceso a mercados }\end{array}$ & Cervantes (2005). \\
internacionales, la infraestructura regional y finalmente con lo & \\
que ocurre dentro de la propia empresa. & \\
\hline La competitividad es la capacidad que posee una & \\
organización para contrarrestar de forma eficiente a sus \\
competidores a través de sus productos o servicios, y poder \\
$\begin{array}{l}\text { acceder al mercado, generando sostenibilidad y aumentar la } \\
\text { participación en el mercado. }\end{array}$ \\
\hline $\begin{array}{l}\text { La competitividad es: la capacidad para competir en los } \\
\text { mercados de bienes y servicios. La capacidad para competir }\end{array}$
\end{tabular}

Fuente: Elaboración propia a partir de los autores citados.

En consecuencia, y analizando los conceptos presentados se puede inferir que la competitividad de una empresa depende de: "La productividad, la rentabilidad, la posición competitiva, la participación en el mercado interno y externo, las relaciones interempresariales, el sector y la infraestructura regional" (García, 2017)

\section{Modelos de evaluación de competitividad empresarial}

Con la caracterización sobre competitividad empresarial, a continuación, se presentan algunos modelos para evaluar la misma. 
Tabla 4. Principales modelos de evaluación de la competitividad empresarial.

\begin{tabular}{|c|c|c|c|}
\hline Modelo & Características & Componentes & Autores \\
\hline 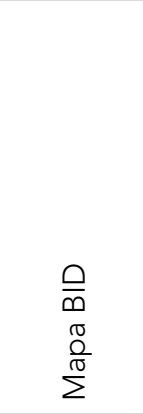 & $\begin{array}{l}\text { Modelo del Banco } \\
\text { Interamericano de } \\
\text { Desarrollo (BID) y } \\
\text { ajustado por Cámara de } \\
\text { Comercio de Medellín } \\
\text { y Antioquia. Es utilizado } \\
\text { para diagnosticar PIMES. }\end{array}$ & $\begin{array}{l}\text { - Planeación estratégica } \\
\text { - Producción y operaciones } \\
\text { - Aseguramiento de la calidad } \\
\text { - Comercialización } \\
\text { - Contabilidad y finanzas } \\
\text { - Recursos humanos } \\
\text { - Gestión ambiental } \\
\text { - Sistemas de información }\end{array}$ & $\begin{array}{l}\text { Martínez y } \\
\text { Álvarez, (2006) }\end{array}$ \\
\hline 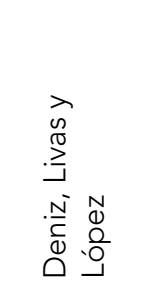 & $\begin{array}{l}\text { Evalúa la competitividad } \\
\text { de empresas del sector } \\
\text { agrícola en el estado } \\
\text { de Colima (México), } \\
\text { toma como referente } \\
\text { el diamante de } \\
\text { competitividad de Porter. }\end{array}$ & $\begin{array}{l}\text { - Estrategia empresarial } \\
\text { - Proceso productivo } \\
\text { - Demanda externa } \\
\text { - Oferta } \\
\text { - Oportunidades y apoyos } \\
\text { institucionales }\end{array}$ & $\begin{array}{l}\text { Deniz, Livas y } \\
\text { López, (2008) }\end{array}$ \\
\hline 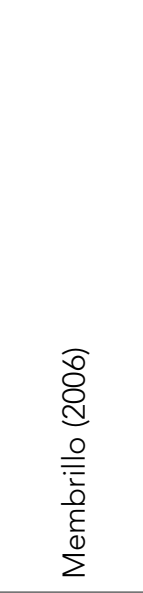 & $\begin{array}{l}\text { Evalúa la competitividad } \\
\text { de empresas del sector } \\
\text { farmacéutico. }\end{array}$ & $\begin{array}{l}\text { - Condiciones dadas: } \\
\text { - Fuerza de trabajo, } \\
\text { - Capital, infraestructura para } \\
\text { investigación. } \\
\text { - Condiciones de demanda y } \\
\text { regulación: } \\
\text { - Mejora de medicamentos } \\
\text { - Regulación de precio } \\
\text { - Investigación y regulación } \\
\text { - Salidas de la industria: } \\
\text { Innovación } \\
\text { Contribución macro económica }\end{array}$ & Membrillo, (2006) \\
\hline $\begin{array}{l}\frac{0}{0} \\
\frac{0}{2} \\
\frac{0}{1}\end{array}$ & $\begin{array}{l}\text { Evalúa la competitividad } \\
\text { de empresas Mexicanas }\end{array}$ & $\begin{array}{l}\text { - Estrategia de negocio } \\
\text { - Recursos humanos } \\
\text { - Administración y organización } \\
\text { - Investigación desarrollo y diseño } \\
\text { - Gestión tecnológica } \\
\text { - Mercadotecnia y servicio } \\
\text { - Finanzas }\end{array}$ & Herrera, (2007) \\
\hline
\end{tabular}

Fuente: Elaboración propia a partir de los autores citados 
Realizando una categorización de los conceptos dados se pueden inferir los siguientes componentes para evaluación de la competitividad organizacional:

- Estrategia.

- Producción.

- Talento humano.

- Gestión organizacional.

- Investigación, innovación y desarrollo.

- Sistemas de información, contable y finanzas.

- Mercadeo y servicios.

- Gestión de medio ambiente

\section{El emprendimiento social y el emprendedor social}

Luego de analizar las empresas sociales y modelos de evaluación organizacional, es importante revisar el concepto de emprendimiento social y las características del emprendedor social para comprender el tipo de liderazgo y gestión que se debería ejercer en empresas con fines sociales, y así desarrollar una propuesta de modelo de competitividad acorde con organizaciones que desarrollan emprendimientos sociales.

\section{El emprendimiento social}

La definición de emprendimiento social continua como un asunto en debate (Harding, 2004), y ha tenido un desarrollo desde entidades sin ánimo de lucro. Por lo anterior, puede presentar fallas en su concepción desde el contexto de su operación teniendo en cuenta las particularidades de los tipos de empresas sociales (mutuales, externas e hibridas)

Para tener un acercamiento a la definición del emprendimiento social, este surge como integración de las siguientes condiciones (Gaiger, 2004): 
- Presencia y reconocimiento en la comunidad de una identidad de trabajo asociativo.

- Existencia de organizaciones que lideran y movilizan a la comunidad.

- Regresión o selectividad del trabajo.

- Deficiencias de las políticas públicas destinadas a generar oportunidades económicas.

- Mediación de organismos externos o de apoyo para generar alternativas de asociatividad.

Es de resaltar que el emprendimiento social’ ${ }^{\circ}$ busca dar soluciones a problemas sociales, generar contribuciones significativas y diversas en sus contextos, territorios o comunidades, para lo cual adopta modelos de negocio que ofrezcan soluciones creativas a los problemas de la comunidad. Por lo tanto, el emprendimiento social: "comprende las actividades y procesos comprometidos a descubrir, definir y aprovechar las oportunidades a fin de aumentar la riqueza social mediante la creación de nuevas empresas o la gestión de las organizaciones existentes de manera innovadora" (Zahra, Gedajlovic, Neubaum, y Shulman, 2009). Bajo este contexto, los emprendimientos sociales son reconocidos por los siguientes elementos (Hoogendoorn, et al., 2010):

- La creación de valor social.

- Tener visión para aprovechar las oportunidades para crear valor social.

- Utilizar la innovación para la adaptación y creación de valor social.

- Suscitan la disposición al riesgo.

- Búsqueda para alcanzar el objetivo social propuesto con los recursos disponibles.

10 Lo "social." en el emprendimiento social ,se construye en alcanzar los propósitos sociales y la generación de valor social; entendido como el aporte al bienestar de una comunidad humana especifica (Dees, 2001) 


\section{Perfil del emprendedor social}

El emprendedor social promueve la generación de impacto social, entendido como: "Un mejoramiento significativo, en algunos casos, perdurable o sustentable en el tiempo, en alguna de las condiciones o características de la población objetivo y que se plantearon como esenciales en la definición del problema que dio origen" y los resultados o beneficios obtenidos por la comunidad se evidencian en:

- Cubrir necesidades de un colectivo determinado (Melián, Camps y Sanchis, 2011).

- Generación de empleo (2011).

- Proveer a la comunidad con los recursos para dar solución a sus problemas particulares (Curto, 2012).

- Proveer herramientas para la solución de problemas comunes (2012).

- Organizar movimientos territoriales para hacer frente a actores más poderosos (2012).

- De otro lado, el emprendedor social debe caracterizarse por una serie de características de tipo aptitudinal como:

- Estructurar organizaciones flexibles tipo horizontal (Melián, Campos y Sanchis, 2011).

- Propagar la innovación social en un porcentaje poblacional importante (Dees, Anderson y WeiSkillern, 2004).

- Fomentar la innovación entre grupos marginados para su uso y así puedan maximizar los activos con los que cuentan (Curto, 2012).

- También debe poseer unas características actitudinales, orientadas a:

- Tener buena relación con el gobierno y sus clientes (Melián, Campos y Sanchis, 2011).

- Robustecer las relaciones entre dentro de los miembros de la organización (Chaves y Sajardo, 2004).

- Dirección participativa, enfatizando el contacto directo entre el empresario y la comunidad intervenida (2004). 
En consecuencia, la competitividad de las organizaciones sociales o de los emprendimientos sociales depende en gran medida del compromiso, valores, actitudes y capacidades aptitudinales de los miembros gestores.

Basado en la naturaleza y propósitos del documento, cuyo objeto centra en el emprendimiento social, se presenta una propuesta de competitividad para la misma.

\section{Modelo de competitividad para el emprendimiento social}

Es de anotar que la competitividad en el emprendimiento social difiere en puntos de fondo con respecto al emprendimiento tradicional o de empresas lucrativas, basado en: las características propias de las empresas sociales, los conceptos de competitividad organizacional, las metodologías de evaluación de la misma, la caracterización del emprendimiento social y el perfil del emprendedor social. Se presentan a continuación los puntos de divergencia.

- El eje misional de los emprendimientos sociales se centra en la generación de valor social y no en la rentabilidad o la productividad.

- Las relaciones interempresariales o con otras organizaciones para los emprendimientos sociales surgen como fuente de apoyo y ayuda para disminuir o aplacar problemas de la comunidad.

- Las características y perfil del talento humano de las organizaciones que integran y gestiona emprendimientos sociales son un factor relevante para alcanzar los propósitos organizacionales.

- La innovación es una estrategia necesaria en el emprendimiento social para buscar soluciones creativas a los problemas sociales y generar un mejor aprovechamiento de los recursos existentes en la comunidad o contexto. 


\section{Por lo anterior se presenta una propuesta de evaluación de emprendimientos sociales, donde se discrimina sus compontes, definición y medios de evalua- ción en la siguiente tabla.}

\section{Tabla 5. Propuesta de evaluación de emprendimiento social con sus compo- nentes.}

\begin{tabular}{|c|c|c|}
\hline Componentes & Definición & Medios de evaluación \\
\hline $\begin{array}{l}\text { Capital social y } \\
\text { administración }\end{array}$ & $\begin{array}{l}\text { Evaluar las relaciones de la } \\
\text { organización para movilizar } \\
\text { recursos en la comunidad } \\
\text { local o intervenida }\end{array}$ & $\begin{array}{l}\text { Cantidad, características y estructura } \\
\text { de las redes de colaboración internas } \\
\text { y externas y su aporte de recursos a la } \\
\text { organización. }\end{array}$ \\
\hline \multirow[t]{2}{*}{$\begin{array}{l}\text { Gestión de } \\
\text { recursos }\end{array}$} & \multirow{2}{*}{$\begin{array}{l}\text { Evaluar el destino de } \\
\text { los recursos para el } \\
\text { cumplimiento del propósito } \\
\text { social. }\end{array}$} & $\begin{array}{l}\text { Características de los gastos operativos, } \\
\text { y su impacto en la comunidad } \\
\text { intervenida o en el producto o servicio } \\
\text { suministrado a la comunidad. }\end{array}$ \\
\hline & & $\begin{array}{l}\text { Aprovechamiento de recursos y fuentes } \\
\text { de financiación. }\end{array}$ \\
\hline Talento humano & $\begin{array}{l}\text { Medir el compromiso de los } \\
\text { individuos que gestionan } \\
\text { la organización con los } \\
\text { propósitos sociales de la } \\
\text { misma. }\end{array}$ & $\begin{array}{l}\text { Consistencia entre la cultura } \\
\text { organizacional y el clima organizacional } \\
\text { frente a los propósitos organizacionales, } \\
\text { y la actitud y aptitudes del } \\
\text { emprendedor social. }\end{array}$ \\
\hline \multirow[t]{2}{*}{$\begin{array}{l}\text { Valor social } \\
\text { y sostenibilidad }\end{array}$} & \multirow{2}{*}{$\begin{array}{l}\text { Evaluar los beneficios } \\
\text { económicos, sociales } \\
\text { y su sostenibilidad en } \\
\text { el contexto, territorio o } \\
\text { comunidad. }\end{array}$} & $\begin{array}{l}\text { Caracterizar los beneficios } \\
\text { suministrados a la comunidad y su } \\
\text { impacto en la disminución o solución } \\
\text { de necesidades o problemáticas en la } \\
\text { comunidad. }\end{array}$ \\
\hline & & $\begin{array}{l}\text { Determinar los aspectos de riesgo y } \\
\text { protección para la sostenibilidad de la } \\
\text { organización. }\end{array}$ \\
\hline \multirow{4}{*}{ Innovación } & $\begin{array}{l}\text { Evaluar las estrategias } \\
\text { implementadas para: }\end{array}$ & \\
\hline & \multirow{2}{*}{$\begin{array}{l}\text { La solución de problemas } \\
\text { de la comunidad o } \\
\text { necesidades de la } \\
\text { comunidad. }\end{array}$} & $\begin{array}{l}\text { Establecer la eficiencia y eficacia de las } \\
\text { estrategias implementadas frente a: }\end{array}$ \\
\hline & & $\begin{array}{l}\text { La problemática o necesidad } \\
\text { identificada }\end{array}$ \\
\hline & $\begin{array}{l}\text { Aprovechamiento de las } \\
\text { oportunidades y recursos } \\
\text { del contexto. }\end{array}$ & Aprovechamientos de los recursos- \\
\hline
\end{tabular}

Fuente: Elaboración propia 


\section{Conclusiones}

Se evidencian diferencias de fondo en los modelos de competitividad para empresas tradicionales, los cuales no abordan aspectos como la creación de capital y valor social.

Se puede inferir que la evaluación de competitividad de emprendimientos sociales debe buscar caracterizar y medir la eficiencia de los mecanismos y redes de apoyo e integración interorganizacional para la generación de valor social

Es relevante que los miembros participantes en la creación y gestión de emprendimientos sociales posean una serie de características orientadas en el trabajo colaborativo con diferentes grupos de interés para la consecución de recursos y la participación activa de la comunidad.

La innovación es, para los emprendimientos sociales, un componente relevante e imprescindible para la sostenibilidad y el aprovechamiento de los recursos existentes en la comunidad.

\section{Referencias}

Bacchiega, A. y Borzaga, C. (2001). Social enterprises as incentive structures. An Economic Analysis. En C. Borzaga and J. Defourny. The Emergence of Social Enterprises. London, UK: Routledge.

Chaves, R. y Sajardo, A. (2004). Economía política de los directivos de las empresas de economía social. CIRIEC-España, 48, 31-52

Cervantes, A. (2005). Competitividad e internacionalización de las pequeñas y medianas empresas mexicanas. Facultad de Ciencias Políticas y Sociales, UNAM. 
Curto, M. (2012). Los emprendedores sociales: innovación al servicio del cambio social. Cuadernos de la Cátedra la Caixa de Responsabilidad Social de la Empresa y Gobierno Corporativo, 13.

Defourny, J. (2001). Introduction. From third sector to social enterprise. En C. Borzaga and J. Defourny. The emergence of social enterprise. London, UK: Routledge.

Defourny, J. (2004). Social Enterprise in an Enlarged Europe: Concept and Realities. Second Conference on Social Economy in the Central and Eastern European Countries: Social Entrepreneurship \& Economic Efficiency. [en línea]. Disponible en: www.emes.net.

Dees, J. G. (2001). The Meaning of " Social Entrepreneurship. Innovation. [en línea]. Disponible en: https://entrepreneurship.duke.edu/news-item/the-meaning-of-socialentrepreneurship/

Dees, J.; Anderson, B. and Wei-Skillern, J. (2004). Scaling Social Impact. Stanford Social Innovation Review, 1(4), 24-32.

Deniz, A.; Livas, J. y López, J. (2008). La competitividad de la micro, pequeña y mediana empresa del sector agrícola exportador del estado de Colima, México. Hitos de Ciencias Económico Administrativas, 14 (38), 29 - 44.

Díaz-Foncea, M. and Marcuello, C. (2012). Social enterprises and social markets: models and new trends. Serv Bus, 6, 61-83.

Gaiger, L. (2004). Emprendimientos económicos solidarios. En A. D. Cattani. (Comp.). La otra economía.

Galera, G. y Borzaga, C. (2009). Social Enterprise: An evolution overview of its conceptual evolution and legal implementation. Social Enterprise Journal, 5 (3), 210228. 
Guzmán y Trujillo, (2008). Emprendimiento Social. Revisión de literatura Estudios Gerenciales, 24(109), 105-16.

Harding, R. (2004). Social enterprise: The ne economic engine? Business Strategy Review, 15(4), 39-43

Herrera, A. (2007). Las fuentes de competitividad en las empresas de software a la medida. Facultad de Contaduría y Administración, UNAM.

Hoogendoorn, B.; Pennings, E. and Thurik, R. (2010). What Do We Know About Social Entrepreneurship: An Analysis of Empirical Research. REPORT SERIES. Policy.

Laville, J. L. and Nyssens, M. (2001). The Social Enterprise, Towards a theoretical socioeconomic approach. En C. Borzaga and J. Defourny. The emergence of social enterprise. London, UK: Routledge.

Mair, J. and Martí, I. (2006). Social entrepreneurship research: A source of explanation, prediction, and delight. Journal of World Business, 41(1), 36-44.

Martínez, J. y Álvarez, C. (2006). Mapa de Competitividad para el diagnóstico de PYMES. $X I$ Foro de Investigación. Congreso Internacional de Contaduría, Administración e Informática. México.

Melián, A.; Campos, V. y Sanchis, J. (2011). Emprendimiento social y empresas de inserción en España. Aplicación del método Delphi para la determinación del perfil del emprendedor y las empresas sociales creadas por emprendedores. Revesco, $106,150-172$

Membrillo, M. (2006). Desarrollo de una propuesta para la evaluación de la relación de actividades innovadoras con la competitividad de empresas farmacéuticas mexicanas. Facultad de Química, UNAM. 
Montoya, L.; Montoya, I. y Castellanos, D. (2008). De la noción de competitividad a las ventajas de la integración empresarial. Revista Facultad de Ciencias Económicas: Investigación y Reflexión, 16(1), 59-70.

Neck, H.; Brush, C. y Allen, E. (2009). The landscape of social entrepreneurship. Business Horizons, 52, 13-19.

Nyssens, M. (2006). Social Enterprise at the crossroads of market, public policy and civil society. En M. Nyssens. (Ed.). Social enterprise: At the crossroads of market, public policies and civil society. London and New York: Routledge.

Palacios, G. (2010). Emprendimiento social: integrando a los excluidos en el ámbito rural. Revista de Ciencias Sociales (RCS), Vol. 16 (4), 579-590.

Pat, G.; Caamal, I. y Avila, J. (2009). Análisis de los niveles y enfoques de la competitividad. Textual (Chapingo), 53, 63-76.

Romo, D., y G, Abdel. (2005). Sobre el concepto de competitividad. Revista de comercio exterior, 55(3), 200-214.

Rubio, L., y V. Baz. (2005). El poder de la competitividad. Centro de Investigación para el Desarrollo. México: A.C. Fondo de cultura económica.

Rubio, A. y Aragón, A. (2006). Competitividad y recursos estratégicos en la Pyme. Revista de empresa, 17, 32-47.

Saavedra, M. (2017). Una propuesta para la determinación de la competitividad en la pyme latinoamericana. Pensamiento y gestión, 33, 93-124.

Salinas, F. y Rubio, M.J. (2001). Tendencias en la evolución de las organizaciones no lucrativas hacia la empresa social. CIRIEC-España, Revista de Economía Pública, Social y Cooperativa, 37, 79-116. 
Spear, R. and Bidet, E. (2005). Social enterprise for work integration in 12 European countries: a descriptive analysis. Annals of Public and Cooperative Economics, 76, $195-231$.

Spear, R.; Cornforth, C. y Aiken, M. (2009). The governance challenges of social enterprises: evidence from a UK empirical study. Annals of Public and Cooperative Economics, 80 (2), 247-273.

Vidal, I. (2001). Spain: Social enterprises as a response to employment policy failure. In C. Borzaga and J. Defourny. The Emergence of Social Enterprises. London, UK: Routledge.

Vidal, I. y Claver, N. (2003). Las empresas sociales en el ámbito de la integración por el trabajo. CIRIEC-España Revista de Economía Pública, Social y Cooperativa, 46, 39-62.

Villajos, E., Sanchis, J.R., y Ribeiro, D. (2012). Empresas Sociales: aproximación empírica a su dirección estratégica. El caso valenciano. CIRIEC-España, Revista de Economía Pública, Social y Cooperativa, 75, 199-221.

Villajos, E. y Sanchis, J. (2013). Las empresas sociales y el management social. Gezki, 10, 189-216.

Zahra, S. A.; Gedajlovic, E.; Neubaum, D. O. and Shulman, J. M. (2009). A typology of social entrepreneurs: Motives, search processes and ethical challenges. Journal of Business Venturing, 24(5), 519-532. 
